Psychotherapeut 2017 -62:306-313

DOI 10.1007/s00278-017-0212-x

Online publiziert: 5. Juli 2017

(c) Der/die Autor(en) 2017. Dieser Artikel ist

eine Open-Access-Publikation.

\section{Redaktion}

T. Storck, Berlin

M. Schouler-Ocak, Berlin

E.-L. Brakemeier, Marburg

CrossMark

\author{
Maggie Schauer ${ }^{1,2} \cdot$ Thomas Elbert $^{1,2} \cdot$ Frank Neuner $^{1,3}$ \\ ' vivo international, NGO \\ ${ }^{2}$ Kompetenzzentrum Psychotraumatologie, Universität Konstanz, Konstanz, Deutschland \\ ${ }^{3}$ Klinische Psychologie und Psychotherapie, Universität Bielefeld, Bielefeld, Deutschland
}

\title{
Narrative Expositionstherapie (NET) für Menschen nach Gewalt und Flucht
}

\section{Ein Einblick in das Verfahren}

Man kann sich einem Trauma nur langsam nähern, mit Sorgfalt, mit Teilnahme ... wenn das Opfer beginnt, sich der Wurzel des Schmerzes zuzuwenden und sich an das erfahrene Leid erinnert. Trauma wird sagbar, weil jemand das Geschehene in eine Geschichte einzuordnen weiß und es so erzählbar macht. Das ist nicht nur eine intellektuelle Herausforderung, sondern auch eine Form moralischer Interaktion mit dem Anderen ... „Alle Sorgen sind zu ertragen, wenn man sie in eine Geschichte packen oder eine Geschichte über sie erzählen kann. Die Geschichte enthüllt die Bedeutung dessen, was sonst eine unerträgliche Folge bloßer Ereignisse bliebe." (S. Benhabib, 23. Oktober 2016, in: Arendt, Menschen in finsteren Zeiten, S. 124 [42])

\section{Hintergrund}

Lebensgeschichten von Menschen nach Migration aus Kriegs- und Krisengebieten zeigen, dass diese häufig soziale, sozioökonomische und traumatische Stressoren erleben mussten (Abbott 2016; Schauer 2016), mit Auswirkungen bis in die nächste oder gar übernächste Generation (Schauer und Schauer 2010; Entringer et al. 2010). Körperliche und soziale Bedrohungen des Menschen und seiner Gruppe (Köbach et al. 2016) führen zu seelischem Leid, sozialen Schmerzen und Verletzungen der moralischen Werte. Bei wiederholtem und zunehmendem Stresserleben sind manifeste klinische Störungen die Folge („Baustein-Effekt“; Schauer et al. 2003): Mit zunehmender, kumulativer Erfahrung von traumatischen Stressoren steigt die Wahrscheinlichkeit von psychischen und auch von chronischen körperlichen Erkrankungen, selbst bei ursprünglich lange Zeit resilienten Individuen (Kolassa et al. 2010; Entringer et al. 2016).

\section{Unrecht, Krankheit und Menschenwürde}

Erfahrung von sozialen Stressoren, Traumata und aggressiven Taten werden behavioral, neuronal und epigenetisch erinnert (Elbert und Schauer 2014). Körper und Gehirn verändern sich dabei in Organisation und Struktur. Neuronal gestützt entsteht ein assoziatives, emotionales Netzwerk an erregenden Verbindungen, das die "heißen“ impliziten Erinnerungen der sinnlich-körperlich-geistigen Erfahrung während des Traumas verknüpft (Elbert und Schauer 2002, 2014). Die damalige peritraumatische Verteidigungsreaktion wird auch in der Gegenwart aktiviert, wenn Erinnerungsreize das Netzwerk an Assoziationen, das sich durch das Erlebnis geformt hatte, erregen. Je häufiger sich Elemente des Erlebens wiederholen, während sich die sensorischen Informationen jeweils (etwas) unterscheiden, umso geringer wird die Anbindung an den jeweiligen Kontext. Raum und Zeitinformationen gehen verloren: Die Netzwerkelemente verschweißen sich gegenseitig, während Zeit und Ort nicht mehr koaktiviert werden können, da unser Gehirn immer nur eine singuläre Aktivierung der Repräsentationen von räumlicher und zeitlicher Vergangenheit zulässt (Elbert et al. 2006): Selbst für die Vorstellung gilt: „Zu einer Zeit kann man nur an einem Ort sein!" So lösen sich die Erinnerungen von ihrer biografischen Zuordnung ab. Ein Gefühl, dem die Vergangenheit fehlt, fühlt sich dann so an, als wäre die Bedrohung gegenwärtig (Schauer et al. 2011). Traumatisierte Menschen sind daher häufig überwältigt von einer hohen Frequenz ungewollt ausgelöster Intrusionen und haben Schwierigkeiten beim intensionalem Zugriff auf das Gedächtnis (Waldhauser et al. 2017), dessen Inhalte raum-zeitlich in den Kontext schlecht strukturiert und inadäquat integriert sind. Durch explizite Kontextualisierung lassen sich emotionale Netzwerkassoziationen schließlich räumlich und zeitlich rückbinden und in die Lebensgeschichte integrieren. In der Folge setzt ein Heilungsmechanismus ein, den sich die Narrative Expositionstherapie (NET) zunutze macht.

Psychotherapie für Geflüchtete muss die soziale und kulturelle Zugehörigkeit der Person aufnehmen sowie seine Lebenslinie und Biografie im Blick haben. Die Erlangung von Würde und die Befriedigung des Wunsches nach Anerkennung des geschehenen Unrechts stellen dabei einen individuellen Beitrag zur kollektiven Bewältigung des Geschehenen dar. Nach Menschen-, Frauen- und Kinderrechtsverletzungen und sozialer 
Erniedrigung kann es in der Therapie nie nur um eine Intervention gehen, die sich nach Traumatisierung den Pathomechanismus "Gedächtnisstörung “ vornimmt - und sei sie noch so treffsicher -, sondern es muss immer um Genesung im echten Sinne gehen, nämlich Rettung, Gesundung und Heimat finden. ${ }^{1}$ Dies beinhaltet Traumaaufarbeitung ebenso wie korrigierende Beziehungserfahrungen im personalen Kontakt, aber auch das Finden von sozialer Zugehörigkeit und Rang, durch Verbesserung der sozialen und beruflichen Funktionsfähigkeit. Die NET wurde insbesondere auch aus diesen Grundsätzen heraus entwickelt (Schauer et al. 2011; Schauer 2015; ter Heide et al. 2016).

\section{Narrative Expositionstherapie}

Die NET stellt einen biografisch-traumaaufarbeitenden Ansatz dar, der dem Überlebenden hilft, wieder Kontrolle über seine eigene Geschichte $\mathrm{zu}$ erlangen. Wie der Name sagt, wird die Lebensgeschichte in ein Narrativ gefasst, und dabei werden emotional hocherregende Szenen der Vergangenheit mithilfe imaginativer Exposition bearbeitet sowie beim Berichten in den chronologischen Zeit- und Geschehensablauf eingebettet. Wir sehen es als Personenrecht an, dass einem Traumaüberlebenden grundsätzlich die Fähigkeit und das Recht zugesprochen werden, zu jeder Zeit qualifiziert zu sein, seine Lebensgeschichte $\mathrm{zu}$ erzählen. $\mathrm{Ob}$ traumatische Inhalte offenbart werden und wann, obliegt dem erzählwilligen Menschen selbst. Nach Klärung der Indikationsstellung (Schauer et al. 2011) können Kinder, Jugendliche sowie erwachsene Personen aller uns bekannter Kulturen an der NET teilnehmen (universell). Der Therapeut sorgt dafür, dass sich der Überlebende sicher und in Kontrolle fühlt.

In der NET werden körperliche und auch soziale Traumatisierungen bearbeitet. Dies erfordert einen Therapeuten mit

\footnotetext{
1 Ahd. Ginesan „nähren“; Wurzel ie. *nes- „heil davonkommen, gerettet werden, überleben";

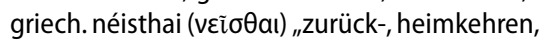
das sichere Heim erreichen" und "gesund werden".
}

Bereitschaft zu empathischem Mitgefühl, Kongruenz im Kontakt und Eintreten für die Menschenrechte („advocacy“), auch um Scham- und Schuldgefühlen entgegenzutreten. Die traumatisierte Person soll ihre Würde wiedererlangen sowie ihre eigene Verantwortlichkeit ausloten können. Im dialogischen Kontakt mit dem Gegenüber und durch einfühlsame Verbalisierung unterstützt (s. unten), wird eine detaillierte und konsistente, schriftliche Narration der Lebensereignisse erstellt („testimony). Die NET hat ein manualisiertes Vorgehen (Schauer et al. 2011; Schauer et al. 2017) und ihre Wirksamkeit kann wissenschaftlich überprüft werden (Evidenzbasierung).

\section{Bearbeitung der Traumata durch Narrative Exposition: praktische Umsetzung}

Versprachlichung durch traumaaufarbeitende Behandlungsverfahren zeigt die höchste Wirksamkeit sowie lang anhaltende Linderung und Heilung von Leiden infolge der traumatischen Stressoren (Schnyder u. Cloitre 2015). Als geeignet hat sich das narrative Einlassen auf den Prozess des willentlichen Aufsuchens der Gedächtnisspuren erwiesen. Die Ordnung der Erinnerungen und die Bearbeitung der Gedächtnispathologie geschehen bei der NET mithilfe klar strukturierter Schritte der Annäherung an das Traumamaterial und haben nicht nur die punktuelle Arbeit am Trauma, sondern immer die Entwicklung des Menschen und die gesamte Lebensgeschichte im Blick.

Im Rahmen der NET wird dem Menschen Gelegenheit gegeben, die stark emotionalen Ereignisse seiner Lebensgeschichte, von der Geburt an, der Reihe nach in einzelnen Episoden zu erzählen. Dies geschieht häufig mithilfe eines Sprachmittlers. Der Therapeut schult den Übersetzer in den Konzepten und Vorgehensweisen der NET (Ruf-Leuschner et al. 2015).

Das klassische Vorgehen der NET (Schauer et al. 2011 [2005]) gliedert sich in folgende 3 Schritte (für Geflüchtete: auch Schauer et al. 2017):
1. Schritt: „Erfassung der Lebensereignisse" (eine bis 2 Doppelsitzungen). Vertikale Abfrage (Ja/Nein Antworten) von möglichen Lebensereignissen nach Typen von Erfahrungen innerhalb eines strukturierten Interviews sowie Bestandsaufnahme der Probleme und Symptome (Komplexitätsgrad gering). Daran knüpft eine Psychoedukation über die Entstehung und Legitimität der Symptome sowie der vorgeschlagenen Intervention an.

2. Schritt: "Lifeline" (eine Doppelsitzung). Horizontale Einordnung der spezifischen Lebensereignisse entlang der Zeitachse in die Lebenszeitperioden (Komplexitätsgrad erhöht: zeitlichräumliche Einbindung in den individuellen Entwicklungszusammenhang). Die Erstellung eines biografischen Überblicks über die hocherregenden Momente (hohes physiologisches Arousal) des Lebens anhand der Lifeline (Schauer und RufLeuschner 2014; Schauer et al. 2014) ist sowohl für traumatisierte Kinder als auch für Erwachsene mit entsprechender Fragmentierung des episodischen Gedächtnisses eine gute Möglichkeit der Reorganisation. Diese raum-zeitliche Zuordnung und überschriftartige Benennung der wichtigsten persönlichen Ereignisse (allgemeine und spezifische Ereignisse) in den aufeinanderfolgenden Lebenszeitperioden sind sinnvolle, aber nicht unabdingbare Bausteine der NET. Bei der Lifeline werden die positiven und die negativen, traurigen und traumatischen Erfahrungen sowie eigene aggressive Taten - wiederum aus der allozentrischen Position auf eine Zeitachse "gelegt": Ein am Boden oder auf dem Tisch liegendes Seil/Schnur symbolisiert häufig den zeitlichen Verlauf der Lebenslinie, auf der dann der Reihe nach biografisch-chronologisch mithilfe von Blumen und Steinen Ereignisse wie Freude/Ressourcen, Traumata, aggressive Akte und spezifische Erlebnisse von Trauer und Verlust symbolisiert werden können (Schauer et al. 2011).

3. Schritt: „Narrative Exposition“ (ca. 6 bis 12 Doppelsitzungen). Chronologische Erzählung der Lebensgeschichte sowie vertieftes und durch Detailabruf 
intensiviertes Erinnern bedeutsamer und traumatischer Lebensereignisse (Komplexitätsgrad hoch: Narrative Exposition und Kontextualisierung der Erfahrungen).

Im Schritt 3 der NET wird das autobiografisch explizite, episodische $\mathrm{Ge}$ dächtnis durch die Narration geformt und vervollständigt. Der Therapeut lädt den Überlebenden ein, seine Lebensgeschichte über mehrere Doppelsitzungen hinweg von seiner Geburt über die ersten Lebensjahre bis zum heutigen Tag zu erzählen (genaue Beschreibung: Schauer et al. 2011 [2005]; Neuner et al. 2013). Dies kann über weite Strecken zusammenfassend geschehen, in Berichten über Situationen, die die Person erlebt hat, sodass der Lebensverlauf deutlich wird. Der Therapeut nimmt dabei die Rolle des einfühlsamen Zuhörers ein. Er istOhrenzeuge für das Geschehen im Leben des Erzählers. Durch sein echtes Interesse und engagiertes Nachfragen befördert und ermuntert er die Narration. Der Therapeut nimmt eine empathische und akzeptierende Haltung ein; er bewertet nicht, was der Mensch fühlt oder denkt; er bewertet allerdings Handlungen, die gegen die Menschenrechte verstoßen (Neuner et al. 2017).

Therapeut oder Beisitzer schreiben die Narration des Überlebenden nieder (für praktische Aspekte dieser Mitschrift: Schauer et al. 2011). In der nächsten Sitzung wird der Text in Ich-Form und wörtlicher Rede vorgelesen, korrigiert und evtl. weitere Details aus der Erinnerung des Überlebenden hinzugefügt. In dieser Weise schreitet die Erzählung voran. Sobald die Narration in die zeitliche Nähe von aufregenden und bedeutsamen Erlebnissen kommt, hilft der Zuhörer, den Bericht detaillierter und tiefer zu fassen. Der Therapeut unterstützt dann das imaginative Wiedererleben, das emotionale Durcharbeiten, die chronologische Einordung der Fragmente. Dies erreicht er v. a. durch das im Folgenden beschriebene Vorgehen.

Verlangsamung. Durch Verlangsamung des Erzählflusses in Momenten hoher physiologischer Erregung soll eine verbesserte Achtsamkeit auf innere Vorgänge gewährleistet werden. In Traumamo-

Psychotherapeut 2017-62:306-313 DOI 10.1007/s00278-017-0212-x

○ Der/die Autor(en) 2017. Dieser Artikel ist eine Open-Access-Publikation.

\section{Schauer $\cdot$ T. Elbert $\cdot$ F. Neuner}

\section{Narrative Expositionstherapie (NET) für Menschen nach Gewalt und Flucht. Ein Einblick in das Verfahren}

\section{Zusammenfassung}

Die Narrative Expositionstherapie (NET) ist ein effizientes Kernelement der Behandlung von Kindern und Erwachsenen, die unter den Folgen von Mehrfach- und Komplextraumatisierung nach Gewalt und Flucht leiden. Die chronologische Erarbeitung einer Narration, der individuellen Lebensgeschichte in der NET ermöglicht Traumaüberlebenden eine Gesamtschau ihres Lebens und so die Integration der Lebenserfahrungen in den biografischen Zusammenhang. Sie aktiviert Ressourcen und erlaubt korrigierende Beziehungserfahrungen im wertschätzenden Kontakt mit einem personalen Gegenüber, der sowohl psychotherapeutisch unterstützt als auch Zeuge des Berichts des Überlebenden ist. Die empathische Begleitung des
Menschen durch ein intensives Wiedererleben während der Versprachlichung der bedeutsamen Lebensereignisse positiver und negativer Valenz führt zur Kontextualisierung von Wahrnehmungen, Gedanken, Gefühlen und Körperempfindungen. Bedeutungen des Geschehens werden in der Distanz zum "traumatischen Damals" erfassbar. Es kommt zur Würdigung der Person und ihrer Geschichte, zur Anerkennung des erlittenen Unrechts.

\section{Schlüsselwörter}

Kriegstrauma · Flüchtlinge · Psychotherapie · Erwachsene $\cdot$ Kinder- und Jugendliche . Narrative Expositionstherapie - NET

\section{Narrative Exposure Therapy (NET) for survivors of violence and forced migration. A glimpse at the intervention}

\section{Abstract}

Narrative Exposure Therapy (NET) provides an effective treatment module for children as well as adults who have suffered from multiple and complex traumatization due to violent interpersonal experiences and forced displacement. The chronological processing of a narration of the individual biography of the refugee through NET enables survivors of traumatic stress to obtain a holistic review of their life and thus the integration of life events into a narrative. This process activates resources and enables corrective relationship experiences by revisiting previous social painful situations together with the therapist. Due to the close and interactive nature of the therapy, attachment repair can occur and shame can be reduced as clients discuss memories without being met with rejection or devaluation. This further assists the development of a positive sense of identity. As an empathic listener, the therapist supports the safe recall or even reliving of the most arousing and meaningful life events, the positive as well as the negative ones. The NET procedure enables recognition of the person and the life history, testifying to human rights violations and regaining dignity.

\section{Keywords}

War trauma - Refugees - Psychotherapy . Adults · Children and adolescents · Narrative Exposure Therapy · NET menten ist das Geschehene in der Regel schnell, überwältigend und verwirrend. Der Therapeut sorgt bei der Erzählung dafür, dass ein sicherer Raum entsteht, um den Vorgang so zu verlangsamen, dass die genaue, dosierte Exploration aller Erlebnisebenen möglich wird.

Vertiefen. Die gezielte Nachfrage ermöglicht eine Vertiefung der Erfahrung während des Erzählens, die den Detailreichtum der Narration erhöht und wiederum das Vorgehen entschleunigt. Ein gutes Verständnis des Geschehens lässt sich durch folgendes Schema des Durcharbeitens erzielen, hier als Vorschlag illustriert:

- Zunächst die Fragen nach der Wahrnehmung: („Was haben Sie wahrgenommen?" „Welche sinnlichen Eindrücke waren da: Sehen, Hören, Riechen, Schmecken, Tasten?").

- Danach die Fragen nach den Gedanken („Als Sie das gehört/gesehen/ 
Hier steht eine Anzeige.

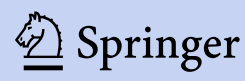




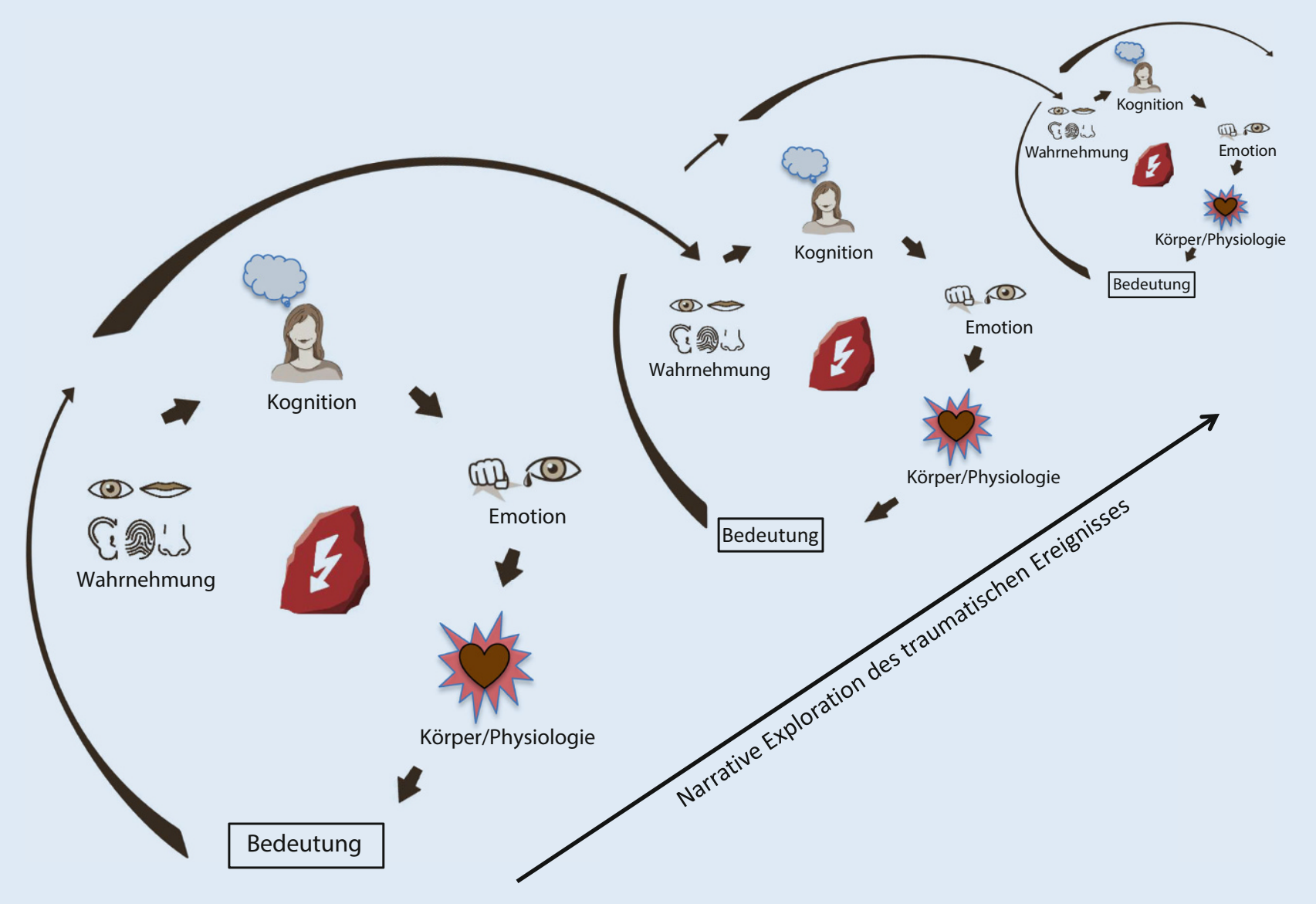

Abb. 1 \ Darstellung der möglichen Dynamik des Erzählprozesses in der Narrativen Expositionstherapie

gefühlt/geschmeckt/gerochen etc. haben, was dachten Sie in diesem Moment?" „Was dachten Sie, was es ist?" „Was ist dann in Ihrem Kopf vorgegangen?" ...).

- Darauf ergeben sich die Fragen nach dem emotionalen und dem körperlichen Erleben („Wie fühlten Sie sich dann in diesem Moment?" „Welche Emotion [Angst, Ekel, Freude, Scham, Wut, Verachtung etc.] entstand, als Sie das wahrnahmen und das dachten?“ „Wie fühlte sich Ihr Körper an?“ „Können Sie dieses Köpergefühl beschreiben?“ „Welche Qualität hat es [heiß, kalt, schwer etc.]?" „Was ist es?" „Wo genau spüren Sie es in Ihrem Körper?" „Was meint es ...").

- Und schließlich die Fragen nach der Bedeutung („Als Sie das wahrgenommen haben und das gedacht und dieses Gefühl dabei hatten, was hat das in diesem Moment für Sie bedeu- tet?“ „Was änderte sich dadurch?“ „Was haben Sie da verstanden?...").

In - Abb. 1 ist die mögliche Dynamik des Erzählprozesses in der NET grafisch dargestellt. Bei der narrativen Exposition hilft der Therapeut dem Patienten, seine Lebensgeschichte zu erzählen und dabei v.a. die aufregendsten Ereignisse langsam, konkret und detailliert auf allen Ebenen des Erlebens darzustellen. Die Narration arbeitet sich nur im traumatischen Moment derart kleinschrittig voran, damit die Sinne, das Denken, die Emotionen, die Körperempfindungen und die Bedeutung all dessen im Zeitlupentempo wahrgenommen werden können. Dadurch hat der traumatisierte Mensch Gelegenheit empathisch begleitet vom Therapeuten - das Geschehene zu verstehen, sein Erleben zu sortieren, die Traumafragmente einzuordnen und explizit in Worte $\mathrm{zu}$ fassen.
Kontrastieren. Während der Überlebende erzählt, hilft der Therapeut, die Traumaebene „Damals“ von der Erzählebene im Hier und Jetzt zu trennen und damit während der Narration den Kontrast zwischen der Traumarealität und der Wiedergabesituation zu erhöhen. Immer wieder geschieht so ein gelenkter Wechsel der Perspektiven zwischen der Erzählung in der Gegenwart und dem Geschehen damals in der Vergangenheit, denn je höher die Gefahr einer vollkommenen Verschmelzung dieser Welten (Flashback) oder der Dissoziationsneigung („shut-down“; Schauer und Elbert 2010; Schalinski et al. 2015; Schauer et al. 2013) ist, umso häufiger lenkt der Therapeut das Bewusstsein des Überlebenden in die Gegenwart (Orientierungsübung) und kehrt erst danach zurück zur Traumaszene. Dies wird als „dual awareness“ bezeichnet: „Merken Sie, dass sie gerade Angst haben, so wie während des ,Traumas. Ich verstehe, dass 


\section{Weiterführende Literatur.}

NET Behandlungsmanual (Original English)

Schauer M, Neuner F, Elbert T (2011) Narrative Exposure Therapy (NET). A Short-Term Intervention for Traumatic Stress Disorders, 2. Aufl. Hogrefe \& Huber, Cambridge Göttingen

Deutsche Fassung (derzeit in Vorbereitung)

Schauer, M., Neuner, F., Elbert, T. (2017) Narrative Expositionstherapie (NET): das Behandlungsmanual. Göttingen: Hogrefe \& Huber Publishers

NET nach Gewalt und Flucht

Schauer M, Elbert T, Neuner F (2017) Narrative Expositionstherapie (NET) nach Gewalt und

Flucht. In: Machleidt W, Heinz A (Hrsg) Praxis der interkulturellen Psychiatrie und Psychotherapie.

Migration und psychische Gesundheit. Elsevier, Urban \& Fischer, im Druck

NET für Erwachsene

Elbert T, Schauer M, Neuner F (2015) Narrative exposure therapy (NET): reorganizing memories of traumatic stress, fear, and violence. In: Schnyder U, Cloitre M (Hrsg) Evidence based treatments for trauma-related psychological disorders. Springer, Berlin Heidelberg New York Tokio, S 229-253

Neuner F, Schauer M, Elbert T (2013) Narrative Exposition. In: Maercker A (Hrsg) Posttraumatische Belastungsstörungen. Springer, Berlin Heidelberg New York Tokio, S 301-350

Schauer M, Ruf-Leuschner M (2014) Lifeline in der Narrativen Expositionstherapie. Psychotherapeut 59:226-238. DOI 10.1007/s00278-014-1041-9

NET für Kinder und Jugendliche (KIDNET)

Schauer, M., Neuner, F., Elbert, T. (2017). Narrative Exposure Therapy for Children and Adolescents (KIDNET). In: M. Landolt, M. Cloitre, U. Schnyder Evidence-Based Treatments for Trauma Related Disorders in Children and Adolescents, pp.227-250, Springer Publishers DOI: 10.1007/978-3-31946138-0_11

Schauer, M., Ruf-Leuschner, M., Landolt, M. (2014) Dem Leben Gestalt geben: Die Lifeline in der Traumatherapie von Kindern und Jugendlichen. In: K. Priebe, A. Dyer (Hrsg.), Metaphern, Geschichten und Symbole in der Traumatherapie, Hogrefe, pp 177-186

NET bei Gewalttätern (FORNET)

Elbert, T., Hermenau, K., Hecker, T., Weierstall, R., Schauer, M. (2012). FORNET: Behandlung von traumatisierten und nicht-traumatisierten Gewalttätern mittels Narrativer Expositionstherapie. In: J. Endrass, A. Rossegger, F. Urbaniok, B. Borchard (Hrsg.) Interventionen bei Gewalt- und Sexualstraftätern: Risk-Management, Methoden und Konzepte der forensischen Therapie. Medizinisch Wissenschaftliche Verlagsgesellschaft, 255-276

Schauer M, Ruf-Leuschner M (2014) Lifeline in der Narrativen Expositionstherapie. Psychotherapeut 59:226-238. DOI 10.1007/s00278-014-1041-9

Sie sich ängstlich fühlen, aber Sie sind hier tatsächlich sicher“. Der Therapeut hilft dies zu überprüfen: „Schauen Sie sich gerne bei mir im Therapieraum/ Behandlungszelt um. Hier kann Ihnen nichts geschehen. Beschreiben Sie mir, wie sich dieser Raum von der Situation damals unterscheidet ..."

Beim Kontrastieren helfen u. a. Hinweise auf:

- raum-zeitliche Unterscheidung (z. B. „Welche Tageszeit war es damals und welche ist es jetzt?“ „Von welcher Seite kam die Sonne damals - und hier im Raum, wo kommt das Licht her?“ „Wie alt warst Du/waren Sie wie lang ist das her?" „Wie lange hat es gedauert?" usw.);

- sensorische Unterscheidungen („Was können Sie hier riechen, wie hat es damals gerochen?" „Beschreiben Sie mir Gegenstände, die in diesem Therapieraum hier sind - damals hat es wie ausgesehen im Raum?“ „Sie sagen, der Atem des Angreifers hat nach Alkohol gerochen, können Sie im Kontrast dazu das Minzöl hier riechen, das Sie in der Hand halten?" usw.);

- Bewusstwerden der Körperhaltung („Merken Sie, wie Sie jetzt während des Erzählens hier auf dem Stuhl sitzen, damals sind Sie im kalten Wasser geschwommen ... tasten Sie ruhig die Armlehnen des Stuhls ab, um sich zu vergewissern“ usw.);

- somästhetische Körpersignale („Damals hat sich der Magen zusammengezogen, ist das heute auch so?" usw.);

- aktive, motorische „countermaneuvers“ der Willkürmuskulatur („Damals wurden Sie ganz schwach und schwer und sind beinahe in Ohnmacht gefallen, können Sie jetzt hier auf dem Stuhl bitte einmal die Wadenmuskulatur ihrer Beine anspannen [Pause], Ihre Handflächen gegeneinander drücken [Pause]“ usw.; detaillierte Beschreibung: Schauer und Elbert 2010).

Diese Vorschläge werden von großem Respekt begleitet und kommen unter Einhaltung der gebotenen Achtung und nur nach vorheriger Psychoedukation zum Einsatz. Der Erzähler soll die Erfahrung von Kompetenz und Kontrolle während der Narration erleben.

Begleiten und empathisch Verbalisieren. Der Therapeut stellt sich innerlich „neben“ den Erzählenden und begleitet ihn raum-zeitlich durch die Szenen seines Lebens. Er stellt sich vor, wie in einem Film das Geschehene bildlich vor sich zu sehen. Der Therapeut spiegelt, verbalisiert Mimik und Gestik und gibt kontinuierlich in eigenen Worten wieder, was er verstanden hat von dem, was die Erzählperson berichtet. Er bemüht sich $\mathrm{zu}$ formulieren, wie es dem Gegenüber geht, was die betroffene Person wohl empfindet, denkt oder auch körperlich erlebt (hat) usw. Er versucht das, was der andere zum Ausdruck bringen will, treffend mit eigenen Worten wiederzugeben und damit sein Verständnis anzubieten. Er hört dem Gesprächspartner während dessen Erzählung mit voller Aufmerksamkeit zu und zeigt seine Einfühlung auch durch Körperhaltung, Mimik und Ausdruckssignale durch körperliche $\mathrm{Zu}$ gewandtheit, Nicken, Blickkontakt. Zum aktiven Zuhören gehören ebenfalls Anstöße zum Weiterreden wie Nachfragen oder das Wiederholen der letzten Worte („Echo") und auch Paraphrasieren (Spiegeln; in eigenen Worten zusammenfassen und wieder anbieten, um $\mathrm{zu}$ kontrollieren, dass alles richtig verstanden wurde und der Zuhörer sein Engagement und Interesse zeigen kann) und Impulse wie: „Und wie ging es dann weiter?"

Die Elemente der als Furchtnetzwerk bezeichneten assoziativen Gedächtnisrepräsentationen werden im Schutz des „Sprechens über" sowie in der Distanz zum Damals aktiviert, sodass die Erfahrungen sich autobiografisch einordnen, benennen, begreifen und verorten lassen. Dies ermöglicht das Eintreten von Erleichterung durch Integration 
und Diskrimination zwischen Trauma und Gegenwart. Durch die empathische und wertschätzende Zuwendung des Gegenübers, die durch ein Verstehen und Einfühlen in die Lebensgeschichte des Patienten möglich wird, macht der Überlebende eine neue, korrigierende Beziehungserfahrung im interpersonalen Kontakt. Während der Aufarbeitung von schmerzhaften Momenten der Biografie, häufig geprägt von emotionaler Deprivation, sozialem Schmerz und Abweisung, verbalisiert der Therapeut die damalige innere Not, benennt empathisch das zurückgewiesene Bedürfnis des verletzten Menschen und bietet therapeutisches Beziehungserleben an. Da die emotionalen Netzwerke der Vergangenheit in der gegenwärtigen Therapie noch einmal zugänglich werden, lassen sich nun auch interpersonale Wunden heilen.

Zusammenfassend lässt sich die Aufgabe des Therapeuten beschreiben als empathische Begleitung und Führung des Überlebenden durch ein intensives Wiedererleben während der Versprachlichung der Lebensereignisse. Letztlich führt dies $\mathrm{zu}$ einem Verstehen alter Wahrnehmungen, Gedanken, Gefühle, Körperempfindungen, während der gleichzeitigen Erlebbarmachung ihrer Bedeutungen in der Distanz zum traumatischen Damals.

Die Erzählung der Lebensgeschichte wird bei der NET mit der frühen Kindheit begonnen und bis zum heutigen Tag geführt sowie schließlich mit Wünschen und Hoffnungen für die Zukunft abgeschlossen.

Wenn möglich, übergibt der Therapeut in der letzten Sitzung die Mitschrift (das Narrativ über die Lebensgeschichte) als gedrucktes Dokument dem Patienten. Sowohl die Zeugen des Berichts (Therapeut, der Dolmetscher und ggf. beisitzender Kotherapeut) als auch der Erzähler (Patient) unterzeichnen das Narrativ (Schauer et al. 2011). Das „Testimony“ kann unterstützend z. B. in das Asylverfahren eigebracht werden und als anonymisierte Version der Sensibilisierung der Öffentlichkeit sowie der kollektiven Vergangenheitsbewältigung dienen.

\section{Evidenz zur Narrativen Expositionstherapie ${ }^{2}$}

Mit therapeutischer Begleitung sich systematisch und chronologisch durch das ganze Leben zu arbeiten, stellt eine robuste Variante effektiver Traumatherapie dar. Ergebnisse von mehr als einem Dutzend systematischer Studien für Erwachsene und Kinder haben die Effektivität von NET zur Verringerung von traumabedingtem psychischem Leid wie posttraumatischer Belastungsstörung, Angststörungen, Depressionen und Komplextrauma nach interpersonaler ebenso wie organisierter Gewalt oder anderen Traumata und Katastrophen nachgewiesen. Wissenschaftliche Übersichtsarbeiten und Metaanalysen beschreiben die Wirksamkeit der Methode (Lely et al. 2017; Tyrer und Fazel 2014; Eberle-Sejari et al. 2015), insbesondere für Überlebende von Gewalt, wobei NET als gut wirksam für Asylbewerber und Geflüchtete angesehen wird (Neuner et al.2017). Gerade auch für Überlebende von Kriegs- und Bürgerkriegserfahrungen hat sich die NET als bedeutsam und hilfreich gezeigt, auch wenn zusätzlich Depression, Schuldgefühle oder gar psychoseähnliche Symptome vorliegen (Schauer et al. 2011). Die NET ist eine gut untersuchte Behandlungsmethode für Überlebende von Folter, wie hohe Effektstärken belegen (Hensel-Dittmann et al. 2011; Neuner et al. 2010; Halvorsen und Stenmark 2010). Deutliche Linderungen des seelischen Leidens ergeben sich in den ersten Monaten nach der Behandlung und gewinnen weiter bis zum Einjahresnachuntersuchungszeitpunkt, was auf eine positive Veränderung der Gesundheit, Funktionalität und Lebensqualität der Patienten schließen lässt. Sie bilden sich auch in der Erholung körperlicher Beeinträchtigungen ab (Morath et al. 2014). Als KIDNET wurde die Narrative Expositionstherapie für Kinder und Jugendliche mit und ohne Eltern sowie auch während ihres Aufenthalts in internationalen Flüchtlingslagern und Flüchtlingsunterkünften erfolgreich zu-

\footnotetext{
2 Hier verweisen die Autoren für die umfangreiche Dokumentation der Literatur aufSchauer et al.(2017).
}

geschnitten und evaluiert (Ruf et al. 2010; Catani et al. 2009; Beiser et al. 2017). Zwei entscheidende Stärken von NET sind die niedrige Drop-out-Rate, d.h., Traumaüberlebende brechen diese Therapie selten ab, und ihr hohes Potenzial für Dissemination, also die Eignung für Schulung und Weitergabe, auch an lokale Helfer in ressourcenarmen Kontexten und in Kriegs- und Krisenregionen (Neuner et al., 2008; Ertl et al. 2011; Jacob et al. 2014; Köbach et al. 2015). Die NET kann also von entsprechend geschulten Traumaberatern als Modul angeboten werden und wäre damit innerhalb einer gesundheitlichen Gesamtversorgung von Geflüchteten in Deutschland denkbar (Elbert et al. 2016).

\section{Fazit für die Praxis}

\section{- Die Narrative Expositionstherapie (NET) ist eine wirksame psychothera- peutische Behandlung für Menschen, die nach Erlebnissen von Gewalt, Flucht und Vertreibung psychisch leiden oder gar funktionsuntüchtig geworden sind. \\ - Die NET ist schlicht in ihrer Durchfüh- rung, da sie auf einem aktiven und einfühlsamen Zuhören bei der chro- nologischen Erzählung von Lebens- ereignissen beruht. Das Verfahren erweist sich als gut disseminierbar und zeigt sich robust in seiner Wir- kung, auch in der Anwendung in ressourcenarmen Kontexten sowie in Situationen von fortdauerndem Stress und Unsicherheit im Lebens- umfeld. \\ - Erwachsene, aber auch Kinder und Jugendliche verschiedenster Kultur- gruppen zeigen hohe Motivation, an einer NET teilzunehmen und diese Behandlung abzuschließen. \\ - Durch das „Testimony“-Element eignet sich die NET zur Menschen- rechtsarbeit.}

\section{Korrespondenzadresse}

\section{PD Dr. M. Schauer}

Kompetenzzentrum Psychotraumatologie, Universität Konstanz

Fach 905, 78457 Konstanz, Deutschland

maggie.schauer@uni-konstanz.de 


\section{Einhaltung ethischer Richtlinien}

Interessenkonflikt. M. Schauer, T. Elbert und F. Neuner geben an, dass kein Interessenkonflikt besteht.

Für die zitierten eigenen Studien gelten die jeweils dort angegebenen ethischen Richtlinien.

Open Access. Dieser Artikel wird unter der Creative Commons Namensnennung 4.0 International Lizenz (http://creativecommons.org/licenses/by/4.0/deed. de) veröffentlicht, welche die Nutzung, Vervielfältigung, Bearbeitung, Verbreitung und Wiedergabe in jeglichem Medium und Format erlaubt, sofern Sie den/die ursprünglichen Autor(en) und die Quelle ordnungsgemäß nennen, einen Link zur Creative Commons Lizenz beifügen und angeben, ob Änderungen vorgenommen wurden.

\section{Literatur}

Abbott A (2016) The troubled minds of migrants. Nature 538(7624):158-160

Arendt H (1989) Menschen in finsteren Zeiten. München, Piper

Beiser M, Mantini A, Srinivasan V, O'Campo P (2017) A Randomized Controlled Trial of Narrative Exposure Therapy (NET) for PTSDAffected Refugee Children and Youth in Canada (submitted)

Catani C, Kohiladevy M, Ruf M, Schauer E, Elbert T, Neuner $F$ (2009) Treating children traumatized by war and tsunami: A comparison between exposure therapy and meditationrelaxation in north-east Sri Lanka. BMC Psychiatry 9:22

Eberle-Sejari R, Nocon A, Rosner R (2015) Zur Wirksamkeit von psychotherapeutischen Interventionen bei jungen Flüchtlingen und Binnenvertriebenen mit posttraumatischen Symptomen ein systematischer Review. Kindh Entwickl 24:156-169

Elbert T, Schauer M (2002) Psychological trauma: Burnt into memory. Nature 419(6910):883-883

Elbert T, Schauer M (2014) Wenn Gegenwartzur Illusion wird. Spuren belastender Lebenserfahrungen in Genom, Gehirn und Geist. Nova Acta Leopold 120(405):3-19

Elbert T, Rockstroh B, Kolassa I-T, Schauer M, Neuner F (2006) The influence of organized violence and terror on brain and mind - a co-constructive perspective. In: Baltes $P$, Reuter-Lorenz $P$, Rösler $F$ (Hrsg) Lifespan development and the brain: the perspective of biocultural co-constructivism. Cambridge University Press, New York, S326-349

Elbert T, Wilker S, Schauer M, Neuner F (2016) Dissemination psychotherapeutischer Module für traumatisierte Geflüchtete: Erkenntnisse aus der Traumaarbeit in Krisen- und Kriegsregionen. Nervenarzt. doi:10.1007/s00115-016-0245-3

Entringer S, Buss C, Wadhwa PD (2010) Prenatal stress and developmental programming of human health and disease risk: concepts and integration of empirical findings. Curr Opin Endocrinol Diabetes Obes 17(6):507

Entringer S, Buss C, Heim C (2016) Early-life stress and vulnerability for disease in later life. Bundesgesundheitsblatt Gesundheitsforschung Gesundheitsschutz 59(10):1255

Ertl V, Pfeiffer A, Schauer E, Elbert T, Neuner $F$ (2011) Community-implemented trauma therapy for former child soldiers in Northern
Uganda: a randomized controlled trial. JAMA 306(5):503-512

Halvorsen JØ, Stenmark H (2010) Narrative exposure therapy for posttraumatic stress disorder in tortured refugees: A preliminary uncontrolled trial. Scand J Psychol 51(6):495-502

Hensel-Dittmann D, Schauer M, Ruf M, Catani C, Odenwald M, Elbert T, Neuner F (2011) The treatment of traumatized victims of war and torture: a randomized controlled comparison of Narrative Exposure Therapy and Stress Inoculation Training. Psychother Psychosom 80:345-352. doi:10.1159/000327253)

Jacob N, Neuner F, Mädl A, Schaal S, Elbert T (2014) Dissemination of psychotherapy for traumaspectrum disorders in resource-poor countries: a randomized controlled trial in Rwanda. Psychother Psychosom 83:354-363

Köbach A, Schaal S, Hecker T, Elbert T (2015) Psychotherapeutic intervention in the demobilization process: addressing combat-related mental injuries with narrative exposure in a first and second dissemination stage. Clin Psychol Psychother. doi:10.1002/cpp.1986

Köbach A, Schauer M, Elbert T (2016) Checklist for Threats to Human Life (THL). Universität Konstanz, Konstanz

Kolassa IT, ErtI V, Eckart C, Kolassa S, Onyut LP, Elbert T (2010) Spontaneous remission from PTSD depends on the number of traumatic event types experienced. Psychol Trauma 2:169-174

Lely JCG, Smid GE, Knipscheer JW, Jongedijk RA Kleber RJ (2017) The Effectiveness of Narrative Exposure Therapy for Traumatised Refugees: A Meta-analysis and Meta-regression Analysis (submitted)

Morath J, Moreno-Villanueva M, Hamuni G, Kolassa S, Ruf-Leuschner M, Schauer M, Bürkle A, Elbert T, Kolassa IT (2014) Effects of psychotherapy on DNA strand break accumulation originating from traumatic stress. Psychother Psychosom 83(5):289-297

Neuner F, Onyut PL, Ertl V, Odenwald M, Schauer E, Elbert T (2008) Treatment of posttraumatic stres disorder by trained lay counselors in an african refugee settlement: a randomized controlled trial.JConsult Clin Psychol 76(4):686-694.doi:10. 1037/0022-006X.76.4.686

Neuner F, Kurreck S, Ruf M, Odenwald M, Elbert T, Schauer M (2010) Can asylum-seekers with posttraumatic stress disorder be successfully treated? A randomized controlled pilot study. Cogn Behav Ther 39:81-91

Neuner F, Schauer M, Elbert T (2013) Narrative Exposition. In: Maercker A (Hrsg) Posttraumatische Belastungsstörungen. Springer, Heidelberg, S301-350

Neuner, Elbert, Schauer (2017) Narrative Exposure Therapy (NET) as a treatment for traumatized refugees. In: Nickerson A, Morina N (Hrsg) Menta health in refugee and post-conflict population. Wiley Blackwell, West Sussex

Ruf M, Schauer M, Neuner F, Catani C, Schauer E, Elbert T (2010) Narrative exposure therapy for 7- to 16-year olds: A randomized controlled trial with traumatized refugee children. J Trauma Stress 23:437-445

Ruf-Leuschner M, Schauer M, Elbert T (2015) Raum für den Dritten: Dolmetscherlnnen in der Therapie von Flüchtlingen, In: Psychotherapie zu Dritt - über die Arbeit mit Dolmetschern in therapeutischen Gesprächen, Sonderheft NTFN. http://www.vivo.org/wp-
content/uploads/2015/05/NTFN-Broschüre S22-28.pdf.Zugegriffen: 27.06.2017

Schalinski I, Schauer M, Elbert T (2015) The shutdown dissociation scale (shut-D). Eur J Psychotraumatol 6:25652. doi:10.3402/ejpt.v6.25652

Schauer M (2015) Narrative exposure therapy. In: Wright JD (Hrsg) International encyclopedia of social \& behavioral sciences, 2. Aufl. Elsevier, Amsterdam, S198-203

Schauer M (2016) The Mass Refugee Movement Better Reframed as Mental Health Crisis?. Global Perspectives of the International Society for Traumatic Stress Studies ISTSS. StressPoints 1. doi:10.13140/RG.2.1.4113.1926

Schauer M, Elbert T (2010) Dissociation following traumatic stress: etiology and treatment. JPsychol 218(2):109-127

Schauer M, Schauer E (2010) Trauma-focused public mental-health interventions: a paradigm shift in humanitarian assistance and aid work. In: Trauma rehabilitation after war and conflict. Springer, New York, S361-430

Schauer M, Schalinski I, Elbert T (2013) Shutdown Dissociationsskala (Shut-D). Universität Konstanz. https://www.zpid.de/pub/tests/PT_9007117_ Shut-D_Fragebogen_englisch.pdf.Zugegriffen: 27.06.2017

Schauer M, Ruf-Leuschner M (2014) Die Lifeline in der Narrativen Expositionstherapie (NET) Psychotherapeut 59:226-238. doi:10.1007/ s00278-014-1041-9

Schauer M, Ruf-Leuschner M, Landolt M (2014) Dem Leben Gestalt geben: Die Lifeline in der Traumatherapie von Kindern und Jugendlichen. In: Priebe K, Dyer A (Hrsg) Metaphern, Geschichten und Symbole in der Traumatherapie. Hogrefe, Göttingen, S177-186

Schauer M, Elbert T, Neuner F (2017) Narrative Expositionstherapie (NET) nach Gewalt und Flucht. In: Machleidt W, Heinz A (Hrsg) Praxis der interkulturellen Psychiatrie und Psychotherapie. Migration und psychische Gesundheit. Urban \& Fischer, Elsevier

Schauer M, NeunerF, ElbertT(2011) Narrative Exposure Therapy (NET). A Short-Term Intervention for Traumatic Stress Disorders, 2. Aufl. Hogrefe \& Huber, Cambridge Göttingen

Schauer M, Neuner F, Karunakara U, Klaschik C, Robert C, Elbert T (2003) PTSD and the "building block" effect of psychological trauma among West Nile Africans. Eur Soc Trauma Stress Stud Bull 10(2):5-6

Schnyder U, Cloitre M (Hrsg) (2015) Evidence based treatments for trauma-related psychologica disorders: A practical guide for clinicians. Springer, Berlin Heidelberg

ter Heide FJJ, Mooren TM, Kleber RJ (2016) Complex PTSD and phased treatment in refugees: $A$ debate piece. Eur J Psychotraumatol 7(1):28687

Tyrer RA, Fazel M (2014) School and communitybased interventions for refugee and asylum seeking children: a systematic review. PLoS ONE 9(2):e89359. doi:10.1371/journal.pone.0089359

Waldhauser GT, Dahl M, Ruf-Leuschner M, MüllerBamouh V, Schauer M, Axmacher N, Elbert T, HansImayr S (2017) Deficient memory control in heavily traumatized refugees (submitted for publication) 